\title{
Pseudogene-mediated posttranscriptional silencing of HMGA1 can result in insulin resistance and type 2 diabetes
}

Eusebio Chiefari', Stefania liritano', Francesco Paonessa', Ilaria Le Pera', Biagio Arcidiacono', Mirella Filocamo², Daniela Foti', Stephen A. Liebhaber ${ }^{3}$ \& Antonio Brunetti, ${ }^{1,4}$

Processed pseudogenes are non-functional copies of normal genes that arise by a process of mRNA retrotransposition. The human genome contains thousands of pseudogenes; however, knowledge regarding their biological role is limited. Previously, we demonstrated that high mobility group A1 (HMGA1) protein regulates the insulin receptor (INSR) gene and that two diabetic patients demonstrated a marked destabilization of HMGA1 mRNA. In this paper we report that this destabilization of HMGA1 mRNA is triggered by enhanced expression of RNA from an HMGA1 pseudogene, HMGA1-p. Targeted knockdown of HMGA1-p mRNA in patient cells results in a reciprocal increase in HMGA1 mRNA stability and expression levels with a parallel correction in cell-surface INSR expression and insulin binding. These data provide evidence for a regulatory role of an expressed pseudogene in humans and establishes a novel mechanistic linkage between pseudogene HMGA1-p expression and type 2 diabetes mellitus.

\footnotetext{
1 Dipartimento di Medicina Sperimentale e Clinica 'G. Salvatore', Università di Catanzaro 'Magna Græcia', viale Europa (Loc. Germaneto), Catanzaro 88100, Italy. ${ }^{2}$ Laboratorio Diagnosi Pre-Postnatale Malattie Metaboliche, Istituto G. Gaslini, Largo G. Gaslini 5, Genova 16147, Italy. ${ }^{3}$ Department of Genetics, University of Pennsylvania School of Medicine, 415 Curie Blvd, Philadelphia, Pennsylvania 19104, USA. ${ }^{4}$ Cattedra di Endocrinologia, Università di Catanzaro 'Magna Græcia', viale Europa (Loc. Germaneto), Catanzaro 88100, Italy. Correspondence and requests for materials should be addressed to A.B. (email: brunetti@unicz.it).
} 
H igh mobility group A1 (HMGA1) is an architectural nuclear protein that binds to AT-rich regions of DNA and functions mainly as a specific cofactor for gene activation ${ }^{1,2}$. As part of an investigation into the molecular basis of regulation of the human INSR gene, we previously identified HMGA1 as a transcriptional regulator specifically needed for proper expression of this gene in eukaryotic mammalian cells ${ }^{3,4}$. Consistent with these findings, human type 2 diabetes (T2D) has been associated with defects in a nuclear regulatory protein either identical to, or highly related to, HMGA1, with commensurate decreases in INSR expression and consequent insulin resistance $\mathrm{e}^{3-5}$

In this study, we report on two unrelated patients affected by insulin resistance and T2D in whom the expression of HMGA1 was markedly reduced and INSR gene transcription was considerably impaired as assessed in Epstein-Barr Virus (EBV)-transformed lymphoblasts of both subjects. Patient 1, a non-obese 45-year-old Italian man with the usual features of T2D, came to medical attention because of fasting hyperglycemia and hyperinsulinemia, in the absence of other disorders such as acromegaly or glucocorticoid excess. Patient 2, an 11-year-old Japanese boy, was diagnosed with $\mathrm{T} 2 \mathrm{D}$ at 6 years of age and had the male form of type A insulin resistance with acanthosis nigricans. By investigating the molecular events underlying the impaired expression of HMGA1 in both diabetic individuals, we have previously identified a heterozygous single-nucleotide $\mathrm{G}$ deletion in the $3^{\prime}$-untranslated region (UTR) of the HMGA1 gene, which seemed to be linked to the accelerated degradation of HMGA1 mRNA in patients' cells ${ }^{5}$. However, when the mutant $3^{\prime}$-UTR was cloned into the $3^{\prime}$ position of a luciferase reporter gene and its expression was analysed in vitro in transient transfection assays, luciferase activity decreased by only $50 \%{ }^{5}$, suggesting that this mutation, although potentially contributing to the loss of HMGA1 mRNA, was not fully sufficient to account for the observed large decline in HMGA1 mRNA stability in vivo in these subjects.

Here, we report the overexpression of a novel HMGA1 pseudogene in both diabetic individuals. This HMGA1-p RNA effectively competes with HMGA1 3'-UTR for a critical RNA stability factor. In this manner, the pseudogene RNA triggers a marked destabilization of HMGA1 mRNA with consequent loss of INSR expression and generation of the insulin resistance phenotype.

\section{Results}

Overexpression of the HMGA1-p pseudogene in diabetic patients. HMGA1 mRNA and protein abundances were determined in EBV-transformed lymphoblasts from diabetic patients and non-diabetic control subjects. In EBV-transformed lymphoblasts from both patients 1 and 2, HMGA1 mRNA and protein levels were markedly reduced compared with levels in nondiabetic controls (Fig. 1a). The decrease in HMGA1 expression in EBV-transformed lymphoblasts from patients 1 and 2 closely paralleled the decrease in INSR mRNA and protein levels. INSR mRNA and protein abundances were normal in EBV-transformed lymphoblasts from all T2D patients expressing normal levels of HMGA1 (Fig. 1a). The protein studies were carried out on nuclear extracts; HMGA1 protein was undetectable in cytoplasmic extracts of patient and control cells. To explore mechanism(s) of HMGA1 deficiency, we carried out studies of HMGA1 mRNA expression by combining conventional and real-time quantitative reverse transcription (RT)-PCR assays with cloning and sequencing strategies. Unexpectedly, the PCR analysis of HMGA1 mRNA revealed two distinct cDNA products (Fig. 1b). Sequencing analyses revealed that the smaller $1,773 \mathrm{bp}$ fragment represented isoform 5 of HMGA1 mRNA, the gene of which mapped on chromosome 6 at $6 \mathrm{p} 21$. In contrast, the $1,873 \mathrm{bp}$ fragment showed $~ 93 \%$ homology with the known sequence of the HMGA1 mRNA and seemed to represent a novel processed pseudogene that we named HMGA1-p.
Its sequence has been deposited in GeneBank (accession number AC005041) as part of chromosome 2, and its position seems to be compatible with a previously identified HMGA1 gene retrotransposed copy ${ }^{6}$. It is to be particularly noted that the level of HMGA1-p mRNA expression was significantly increased in both patients when compared with healthy controls and with 27 of unrelated T2D subjects with and without the G deletion above (including the two previously identified patients with a hemizygous deletion of the HMGA1 gene ${ }^{5}$ ), whereas mRNA abundance of its homologous HMGA1 coding gene was markedly reduced (Fig. 1b). Moreover, as revealed by northern blot analysis of total RNA from cultured lymphoblasts of patients and controls, the HMGA1/ HMGA1-p molecular ratio was considerably decreased in patients 1 and 2 (Fig. 1b).

HMGA1-p affects HMGA1 mRNA and INSR expression and function. The reciprocal relationship between $H M G A 1$ and $H M G A 1-p$

a
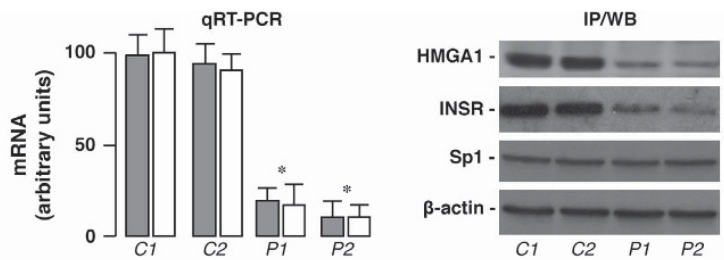

b
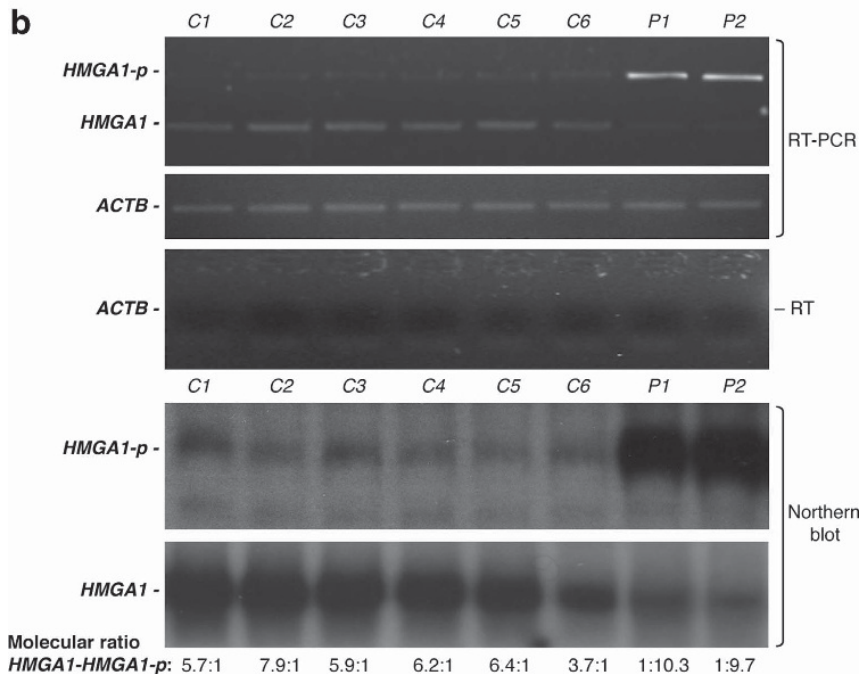

Figure 1 | HMGA1, HMGA1-p and INSR expression levels. (a) Comparison of HMGA1 mRNA (grey bars) and protein content with INSR mRNA (white bars) and protein expression in cultured lymphoblasts from controls $(C 1, C 2)$ and patients (P1, P2). C1, representative of 20 normal subjects; $\mathrm{C} 2$, representative of 20 T2D patients with normal INSR expression. Normalized mRNA is expressed as a percentage of maximal value (100\%). Results are the mean \pm s.e.m. of three separate assays. ${ }^{\star} P<0.001$ compared with controls. IP of the INSR and WB were performed using an anti-INSR $\beta$-subunit antibody. Sp1 and $\beta$-actin, control of nuclear and total cellular protein loading, respectively. (b) HMGA1 and HMGA1-p mRNA as detected by a unique set of primers in semiquantitative RT-PCR of cDNA from cultured lymphoblasts of patients (P1, P2) and non-diabetic (C1-3, representative of 20 healthy subjects) and diabetic (C4-5, representative of 20 T2D patients) controls. mRNAs from T2D patients with the same $\mathrm{G}$ deletion as $\mathrm{P} 1$ and $\mathrm{P} 2$, but without HMGA1-p overexpression (C6, representative of seven T2D patients) are shown. Values were standardized with ACTB mRNA. Genomic DNA contamination in RNA samples was excluded by PCR using aliquots of RNA without reverse transcription ( - RT). Total RNA $(5 \mu \mathrm{g})$ from the same set of cultured lymphoblasts was analysed by northern blot using cDNA probes for HMGA1 and HMGA1-p. Representative autoradiograms are shown. 
mRNA suggested that overexpression of the HMGA1-p pseudogene may result in decreased HMGA1 protein expression, thereby leading to the observed decrease in INSR expression and the diabetic phenotype in these individuals. To test this model, a recombinant plasmid carrying the entire HMGA1- $p$ pseudogene was generated and transiently transfected into cultured human HeLa cells, a cell line naturally expressing the homologous HMGA1 coding gene. As shown in Fig. 2a, treatment of HeLa cells with increasing amounts of HMGA1-p-containing plasmid caused a dose-dependent decrease in HMGA1 mRNA levels, with the maximal inhibition (50-60\%) occurring at $2 \mu \mathrm{g}$ recombinant vector. This reduction was specific for $H M G A 1$, as mRNA expression for the ubiquitously expressed transcription factor Sp1 was unaffected. Reduced HMGA1 mRNA levels paralleled the decrease in HMGA1 protein levels as detected by western blot (WB) analysis of nuclear extracts from transfected HeLa cells (Fig. 2a). Similar results were obtained in human HEK293 cells, which do not express appreciable levels of endogenous HMGA1. As shown in Fig. 2a, HMGA1 mRNA and protein levels were impaired in HEK-293 cells when both HMGA1 and HMGA1-p were coexpressed simultaneously. When forced expression of
HMGA1- $p$ was induced in 3T3-L1 adipocytes (and in other insulinsensitive human and mouse cell lines, such as HepG2 and Hepa-1 liver cell lines), HMGA1 and INSR expression levels were reduced, and this reduction was associated with a decrease in insulin signalling events, such as insulin-stimulated INSR $\beta$-subunit autophosphorylation and the phosphorylation of the insulin receptor substrate 1 (IRS1) (Fig. 2b).

Accumulating evidence strongly implicates posttranscriptional mechanisms involving mRNA structure (for example, folding) and mRNA stability in the regulation of gene expression ${ }^{7}$. To determine whether HMGA1-p overexpression in patients 1 and 2 was involved in posttranscriptional processes affecting HMGA1 expression, we examined the half-life of HMGA1 mRNA in the EBV-transformed lymphoblasts of patients. HMGA1 mRNA half-life decreased from $\sim 26 \mathrm{~h}$ in control cells to $4-6 \mathrm{~h}$ in cells of diabetic individuals overexpressing HMGA1-p (Fig. 2c). When studies of mRNA decay were conducted in patients' cells transfected with small interfering RNA (siRNA) specific to HMGA1-p, HMGA1 mRNA was stabilized, exhibiting a half-life of 10-12 h (Fig. 2c), along with a parallel increase in HMGA1 mRNA abundance (Fig. 2d). These data

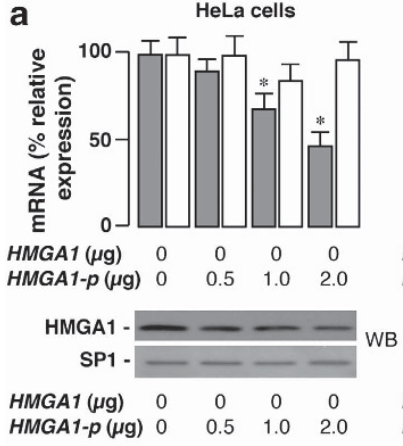

b

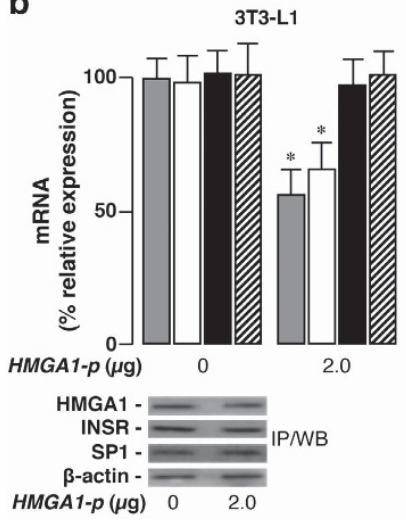

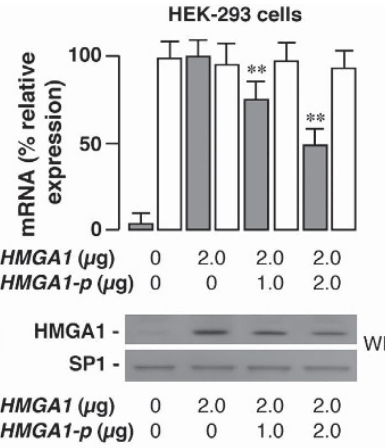

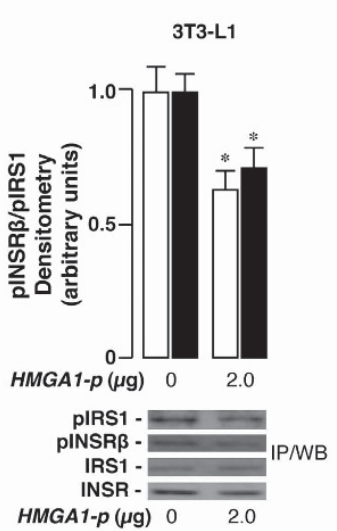

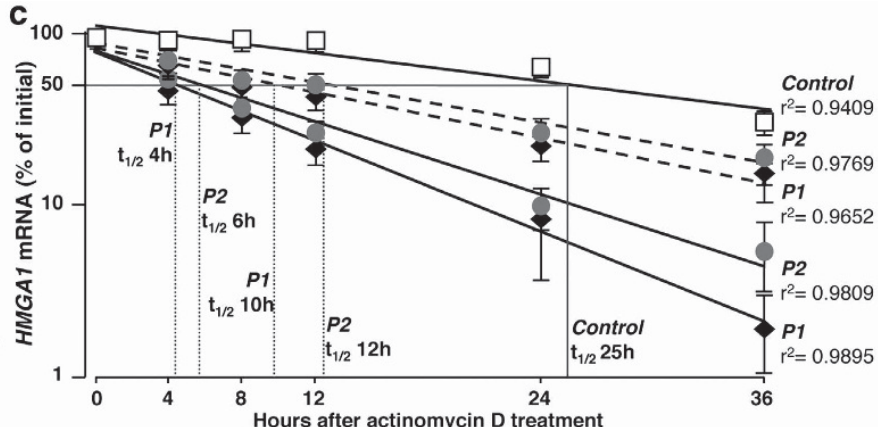

d
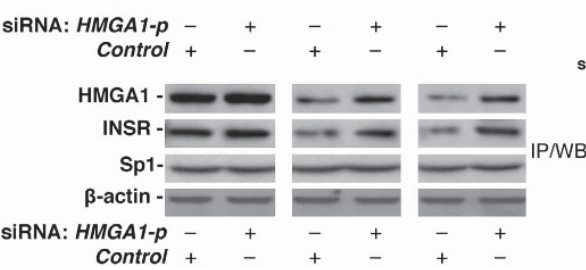

e

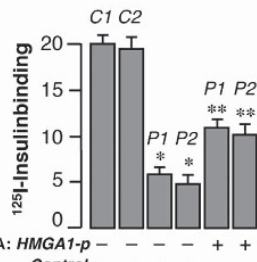

Control +++++

Figure 2 | Impact of HMGA1-p on HMGA1 mRNA levels and stability and on INSR expression and function. (a) HeLa and HEK-293 cells were transfected as indicated, and HMGA1 (grey bars) and control Sp1 (white bars) mRNA and protein levels were measured by real-time PCR and WB, respectively. mRNA is expressed as a percentage of max (100\%, untransfected HeLa cells or HEK-293 cells plus HMGA1 alone). Results are the mean \pm s.e.m. of three experiments. ${ }^{\star} P<0.05$ versus untransfected HeLa cells; ${ }^{\star \star} P<0.05$ versus HEK-293 cells overexpressing HMGA1 alone. (b) Left: HMGA1 (grey bars), INSR (white bars), Sp1 (black bars) and $\beta$-actin (slashed bars) mRNA and protein (IP/WB), as measured in 3T3-L1 adipocytes, before and after transfecting HMGA1-p expression vector. Right: INSR and IRS1 expression and INSR $\beta$-subunit (pINSR $\beta$ ) and IRS1 (pIRS1) phosphorylation were determined by IP/WB of insulin-stimulated lysates from untransfected and transfected cells. White bars, pINSRß; black bars, pIRS1. Densitometric results are the mean \pm s.e.m. Results are from three independent experiments. ${ }^{\star} P<0.05$ versus untransfected 3T3-L1 cells. (c) HMGA1 mRNA decay in patients and control cells treated with non-targeting siRNA (solid lines), or HMGA1-p siRNA (dashed lines). Open square, control (representative of 20 normal subjects); solid diamond, patient 1 (P1); solid circle, patient 2 (P2). mRNA amount at each time point is expressed as a percentage of max (100\%, time 0), plotted on semilog axes. Results are the mean \pm s.e.m. of triplicates from three independent experiments. (d) HMGA1-p (black bars), HMGA1 (grey bars) and INSR (white bars) mRNA in cultured lymphoblasts from controls $(n=20)$ and patients 1 and 2, after treatment with control/HMGA1-p siRNA. Results are means \pm s.e.m. of three real-time PCRs. ${ }^{*} P<0.05$ versus untreated cells, per condition. HMGA1 and INSR protein levels are shown in representative IP/WB of three independent experiments. (e) Insulin binding to EBV-transformed lymphoblasts pretreated with control/HMGA1-p siRNA. Binding is expressed as percentage of total $/ 10^{7}$ cells. Results are the mean \pm s.e.m. of three separate experiments. C1, healthy subjects $(n=20) ; C 2$, T2D patients with normal HMGA1 expression ( $n=20)$; P1 and P2, patients 1 and $2 .{ }^{\star} P<0.001$ versus control $(\mathrm{C} 1, \mathrm{C} 2)$ cells; ${ }^{\star \star} P<0.05$ versus untreated patient's $(\mathrm{P} 1, \mathrm{P} 2)$ cells. 
revealed an inverse correlation between $H M G A 1-p$ pseudogene expression and expression of its homologous HMGA1 coding gene in these individuals. Interestingly, the partial restoration of $H M G A 1$ mRNA levels after treating cells with siRNA against HMGA1-p was accompanied by a concomitant increase in INSR mRNA and protein expression levels (Fig. 2d), and this increase was followed by a restoration of cell-surface insulin binding in patients' cells (Fig. 2e). The fact that transfection of patients' cells with siRNA to HMGA1-p significantly, but not completely, corrected HMGA1 mRNA stability and levels is consistent with the possibility that G deletion in the $3^{\prime}$-UTR of the HMGA1 gene, in these patients, can synergize with HMGA1-p overexpression in inducing HMGA1 mRNA destabilization/degradation.

Impact of HMGA1-p $3^{\prime}$-UTR on HMGA1 mRNA expression. To further explore the role of HMGA1-p in the control of $H M G A 1$ mRNA stability, we mapped the segment of the HMGA1- $p$ RNA that is critical to this pathway. Cells were transiently transfected with recombinant vectors carrying either the full-length or the truncated version of HMGA1-p. As shown in Figure 3a, transfection of HeLa cells with the recombinant plasmid HMGA1-p lacking the $248 \mathrm{bp} 5^{\prime}$ region (corresponding to the $5^{\prime}$-UTR of HMGA1 gene) caused a $50 \%$ decrease in HMGA1 mRNA expression. This reduction was similar to the reduction obtained by transfecting fulllength HMGA1-p. In contrast, no decrease in HMGA1 expression was observed in cells transfected with plasmid containing HMGA1$p$ lacking the $1,276 \mathrm{bp} 3^{\prime}$ region (corresponding to the $3^{\prime}$-UTR of the HMGA1 gene) (Fig. 3a), suggesting that cis-acting element(s) in the $3^{\prime}$ region of HMGA1-p is (are) responsible for the observed impact on HMGA1 mRNA instability/degradation. This observation is consistent with our previous results indicating that HMGA1 $3^{\prime}$-UTR has critical posttranscriptional regulatory elements within its $3^{\prime}$-UTR ${ }^{8}$. To characterize in greater detail those regions of the HMGA1-p 3'-UTR that were implicated in posttranscriptional processes affecting HMGA1 mRNA stability/degradation, several deletion mutants of HMGA1-p 3'-UTR, containing homologous regions of $H M G A 1$, were cloned into the pcDNA 3 plasmid vector, and analysed in transient transfection assays for their ability to interfere with transcription of the homologous HMGA1 gene. Realtime RT-PCR analysis revealed that the expression level of endogenous HMGA1 was only slightly increased in HeLa cells, in the presence

\begin{tabular}{|l|l|l|l|l|l|}
\hline \\
\hline
\end{tabular}

Figure 3 | Effects of HMGA1-p and its derivatives on HMGA1

expression. (a) HMGA1 mRNA was reduced in HeLa cells transfected with expression vectors containing the homologous HMGAT-p 3'-UTR (grey bar) or the full-length HMGA1-p (black bar), and was unaffected in cells transfected with the expression vector containing the homologous HMGA1-p 5'-UTR (dashed bar). The relative activity of $p G L 3$ control vector alone was set at $100 \%$. Results are the mean \pm s.e.m. of three independent real-time PCRs. $P<0.05$ versus control. (b) Left: schematic representation of HMGA1 $3^{\prime}$-UTR and various deletion mutants of the HMGA1-p homologous region. Highly conserved regions (1-4) are highlighted in both 3'-UTR regions. Right: Effects of deletion mutants on HMGA1 mRNA expression, as measured by real-time PCR in transfected HeLa cells. Results are the mean \pm s.e.m. of three separate experiments. of recombinant plasmids bearing proximal deletion mutants of HMGA1-p 3'-UTR ( $\Delta 291-\Delta 1026)$ (Fig. 3b). In contrast, HMGA1 mRNA abundance was considerably decreased in the presence of recombinant constructs containing the distal deletion mutants of HMGA1-p 3'-UTR ( $\Delta 1253$ and $\Delta 1276)$ (Fig. 3 b). As shown in Fig. $3 b$, deletion of $152 \mathrm{bp}$ of the $3^{\prime}$ end of the HMGA1-p $3^{\prime}$-UTR (region 4) reduced HMGA1 expression to approximately $40-50 \%$, suggesting the presence, within this region, of elements that can decrease endogenous HMGA1 mRNA expression in trans. A possible mechanism for this control may be titration by HMGA1-p RNA of one or more trans-acting cytoplasmic protein factors critical to HMGA1 mRNA stability.

Physical association of $\alpha \mathrm{CP} 1$ with the HMGA1 and HMGA1-p $3^{\prime}$ UTR. To better understand the mechanism underlying the ability of $H M G A 1-p$ to reduce HMGA1 mRNA stability and induce HMGA1 mRNA degradation, we carried out a series of RNA-electrophoretic mobility shift assay (EMSA) combined with pull-down and immunoblot assays to identify the corresponding shared RNA-binding proteins. As shown in Figure 4a, RNA-protein binding activity was detected with both HMGA1 (H7) and HMGA1-p (H7-p) biotinylated 3 -UTR mRNA probes, in the presence of increasing amounts of cytoplasmic extracts from HeLa cells. To determine the specificity of RNA-protein binding, competition assays were performed. A 30-fold excess of either unlabelled H7 or unlabelled H7-p significantly reduced the binding of labelled $\mathrm{H} 7$ and labelled $\mathrm{H} 7-\mathrm{p}$ to the RNA-binding proteins, respectively, whereas the presence of the same amount of nonspecific competitor RNA had no significant effect on the complex formation (Fig. 4a). Moreover, unlabelled H7-p competitively inhibited the binding of labelled H7 to cytoplasmic proteins (Fig. 4a). In concert with this observation, unlabelled H7 inhibited the binding of labelled H7-p to cytoplasmic proteins (Fig. 4a). As this region of $3^{\prime}$-UTR mRNA contains three prominent and potentially important C-rich stretches, additional competition experiments were carried out with the synthetic homoribopolymer poly $(\mathrm{C})$. Interaction of HeLa cytoplasmic proteins with either H7 or H7-p was markedly decreased in the presence of increasing amounts of poly(C) (Fig. 4b). This effect was specific, as no appreciable interference was detected in the presence of homoribopolymers poly $(A)$, poly $(G)$ or poly $(U)$, and this specificity was further confirmed by lack of RNA-protein binding on incubation with the H7 probe in which mutations within the $\mathrm{C}$-rich stretches were generated (Fig. 4b). On the basis of these observations, we hypothesized a role in RNA-protein binding activity for $\alpha \mathrm{CP}$ proteins, a group of $\mathrm{KH}$-domain RNA-binding proteins with binding specificity to C-rich patches, which have prominent roles in mRNA stability control $^{1-11}$. Consistent with this hypothesis, the unique protein-RNA complex in HeLa cells was recognized and supershifted to a slowermigrating form by incubation with an affinity purified antiserum to the C-binding protein, $\alpha \mathrm{CP} 1$ (FF1) (Fig. 4c). These data were substantiated by pull-down and RNA immunoprecipitation (IP) of $\alpha \mathrm{CP} 1-\mathrm{RNA}$ complexes from HeLa cell extracts (Fig. 4d), suggesting that $\alpha \mathrm{CP} 1$ binds in vivo, as well as in vitro, to HMGA1 and HMGA1$p$ RNAs. In agreement with these observations, HMGA1 mRNA and protein levels were decreased in HeLa cells following siRNA-mediated knockdown of endogenous $\alpha \mathrm{CP} 1$ expression (Fig. 4e).

Thus, we propose that by competing for the trans-acting cytoplasmic protein, $\alpha \mathrm{CP} 1$, the overexpression of the HMGA1-p pseudogene RNA in affected individuals destabilizes HMGA1 mRNA with a corresponding decrease in HMGA1 protein production (Fig. 5). The decrease in HMGA1 results in a corresponding decrease in INSR gene transcription, with consequent insulin resistance and T2D.

\section{Discussion}

Processed pseudogenes result from reverse transcribed mRNAs ${ }^{12}$. In general, because pseudogenes lack promoters, they are no longer 
a H7
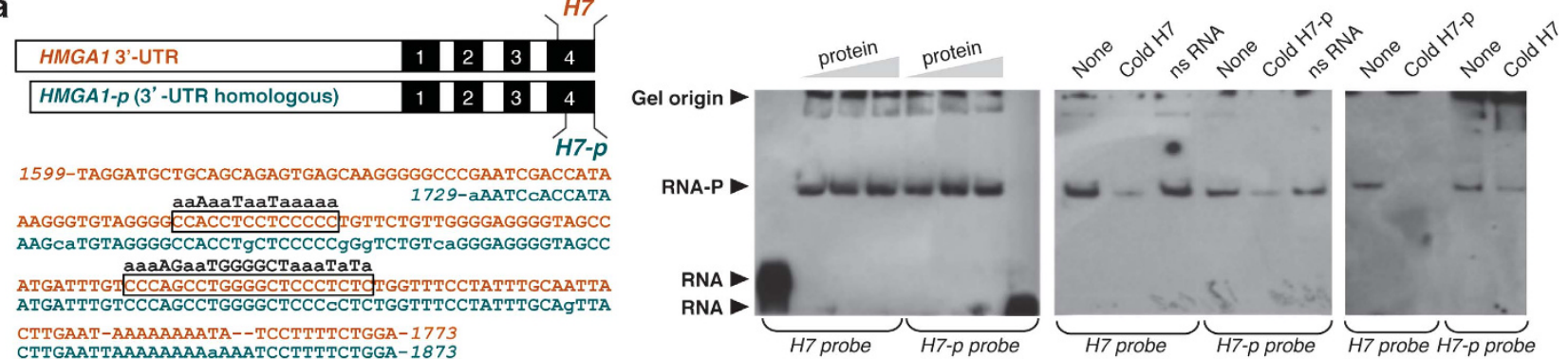

b

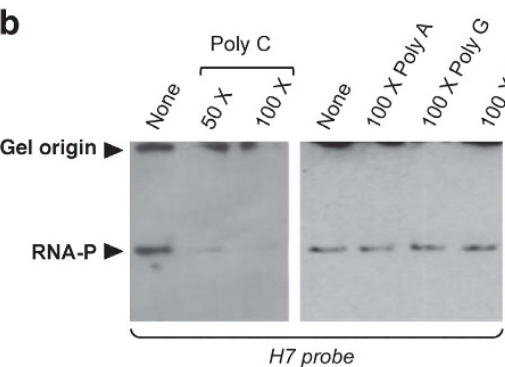

H7 probe

d

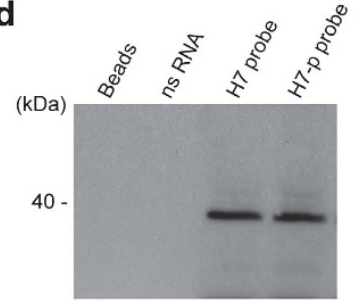

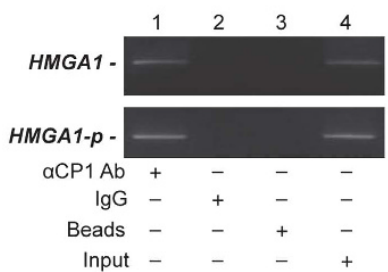

e

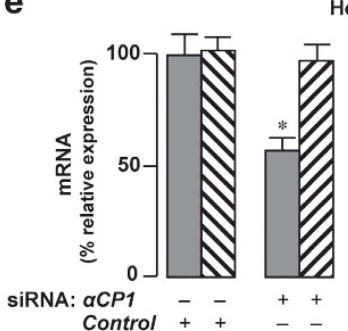

c

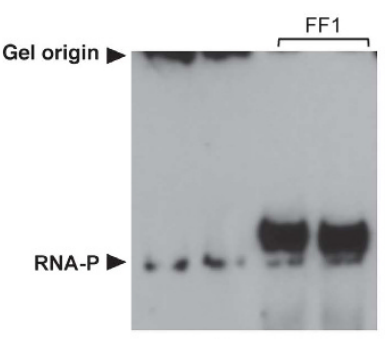

H7 probe

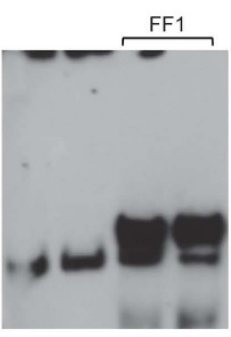

H7-p probe

HeLa cells

Figure 4 | mRNA-protein binding activity, physical association of $\alpha$ CP1 with HMGA1 and HMGA1-p 3'-UTR and $\alpha$ CP1 depletion. (a) Left: Localization and cDNA sequences of probes H7 (brown) and H7-p (green). C-rich elements targeting $\alpha \mathrm{CP} 1$ are in boxes. Mutated C-rich elements of H7 are in black lowercase letters. Right: RNA-EMSAs showing binding of the $\alpha$-complex family member(s) to either probe $\mathrm{H} 7$ or H7-p. HeLa protein extracts (2-6 $\mu$ g, left panel) were incubated with either probe, and complexes resolved by EMSA. Sequence specificity of RNA-binding proteins was determined by using a 30-fold molar excess of unlabelled H7, H7-p or a nonspecific (ns) competitor RNA (centre panel). Labelled H7 or H7-p RNA was incubated with protein, in the absence or presence of a 30 -fold molar excess of unlabelled $\mathrm{H} 7$ - $\mathrm{p}$ or unlabelled $\mathrm{H7}$, respectively, and cross-competition of $\mathrm{H} 7$ and $\mathrm{H} 7$ - $\mathrm{p}$ was analysed (right panel). Free (RNA) and bound (RNA-P) probes are indicated in representative assays. (b) Effect of homoribopolymers poly(C), poly(A), poly $(\mathrm{G})$, poly $(\mathrm{U})$ and a mutated $\mathrm{H} 7(\mathrm{mH} 7)$ probe on EMSA. (c) In supershift assays, extracts were preincubated with the anti- $\alpha \mathrm{CP} 1$ antibody (FF1 antiserum) before adding the probe. (d) RNA pull-down and RNA IP. Lysates were subjected to pull-down (left) in the presence of biotinylated H7, H7-p or nonspecific (ns) RNA probe, followed by WB with FF1 antiserum. RT-PCR was used for the detection of HMGA1 and HMGA1-p mRNA (right) in HeLa extracts following IP of $\alpha$ CP1 protein, using the FF1 antiserum. Co-immunoprecipitated RNA, as well as RNA present in an aliquot of the initial extracts, was subjected to RT-PCR (input). IP using rabbit IgG, used as negative control. (e) Effects of $\alpha C P 1$ depletion. HeLa cells were treated with anti- $\alpha C P 1$ siRNA (100 pmol) or a non-targeting control siRNA, and endogenous HMGA1 mRNA (grey bars) was measured 48-96 h later by quantitative RT-PCR. In parallel experiments, HMGA1 protein expression was revealed by WB from HeLa extracts. Lamin A/C mRNA (dashed bars) and protein, used as controls. Representative WB is shown. Densitometric analyses of $\alpha$ CP1 (white bars) and HMGA1 (grey bars) WB are in bar graphs. Results are the mean \pm s.e.m. of four separate assays. ${ }^{\star} P<0.05, H M G A 1$ mRNA versus siRNA control; ${ }^{* \star} P<0.05, \alpha C P 1$ and $H M G A 1$ protein levels versus siRNA control.

functional from the moment they are inserted into the genome and are not transcribed into $\mathrm{mRNA}^{13-18}$. However, recent evidence indicates that a subset of processed pseudogenes may perform regulatory functions. The ENCODE project has estimated that $\sim 20 \%$ of pseudogenes are transcribed with a pattern of tissue or cell line specificity ${ }^{18,19}$, and a number of functional pseudogenes have been shown to be indispensable for the evolution of mammals and can affect gene expression ${ }^{20,21}$. For example, it has been shown that, in Lymnea stagnalis, a pseudogene homologous to the nitric oxide synthase gene decreases the expression levels of his normal gene of origin through the formation of an RNA duplex that arises through a reverse-complement sequence found at the $5^{\prime}$ end of the pseudogene transcript ${ }^{22}$. In a second example, transcription of the makorin1-p1 pseudogene in mouse was required for the stability of mRNA from a homologous gene makorin $1^{23}$. Notwithstanding, this latter study has been subsequently challenged ${ }^{24}$, and a biological function for transcribed pseudogenes has been confirmed in several successive studies ${ }^{25-30}$. Despite multiple lines of evidence supporting functional role(s) of pseudogenes in mammals, evidence of a role of such interactions in human disease has heretofore been lacking.

In this study, we describe a novel human HMGA1 pseudogene, HMGA1-p, which regulates mRNA stability of its corresponding coding gene. The data are consistent with a model by which the HMGA1-p pseudogene transcript effectively competes for a transacting cytoplasmic protein critical to the longevity of HMGA1 mRNA. By accelerating the degradation of HMGA1 mRNA transcript, the elevated levels of HMGA1- $p$ RNA in affected individuals suppress expression of the INSR gene, resulting in insulin resistance and T2D. Previous studies have demonstrated that HMGA1 gene expression is posttranscriptionally regulated through signals 

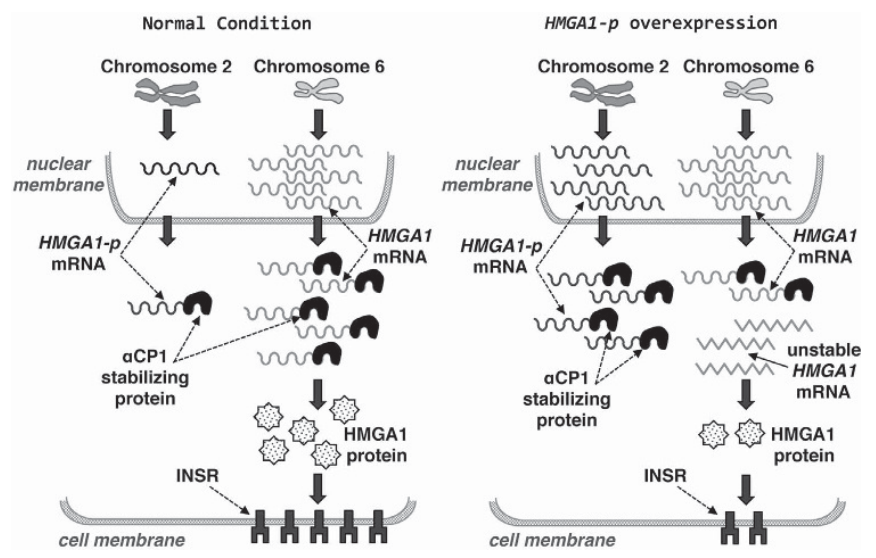

Figure 5 | Model for the role of HMGA1-p pseudogene in diabetes. In

the two individuals described in this report, abnormally high levels of the HMGA1-p pseudogene transcript are shown to compete with HMGA1 mRNA for the RNA-binding protein $\alpha C P 1 . \alpha C P 1$ normally stabilizes HMGA1 mRNA on binding to a C-rich stability determinant in its $3^{\prime}$-UTR. By accelerating the degradation of HMGA1 mRNA, HMGA-1p RNA inhibits HMGA1 protein production with a consequent decrease in INSR gene transcription. The loss of INSR protein underlies the resultant insulin resistance and $\mathrm{T} 2 \mathrm{D}$ in these two individuals.

within its $3^{\prime}$-UTR. Four highly conserved regions in this region seem to provide target-binding sites for RNA-binding proteins that are capable of either activating or inhibiting HMGA1 protein production $^{8}$. Consistent with this observation, here we show that the ability of HMGA1-p to destabilize HMGA1 mRNA maps to the $3^{\prime}$ terminal portion of HMGA1-p mRNA. Remarkably, this region, which maintains extensive sequence homology with the HMGA 13 '-UTR, contains a series of three C-rich motifs. Such $\mathrm{C}$-rich repeats have been previously linked to stabilization of a number of long-lived mRNAs ${ }^{31,32}$. By performing RNA-protein binding studies, we demonstrated that $\alpha \mathrm{CP} 1$ interacts with either HMGA1 or HMGA1-p 3'-UTR and that cross-competition between the two RNA species for $\alpha \mathrm{CP} 1$ can occur in both human and murine cells, in which increased levels of HMGA1-p transcript, by competing for the same trans-acting element involved in mRNA stabilization, may lead to increased HMGA1 mRNA degradation. The detrimental impact of HMGA1-p overexpression on HMGA1 mRNA expression was substantiated by siRNA knockdown of HMGA1- $p$ in patient-derived lymphoblasts, in which the reduction in HMGA1-p transcript abundance was followed by a sustained increase in HMGA1 mRNA stability with an improvement in INSR expression and insulin-binding capacity. As $\alpha \mathrm{CP} 1$ binds to many mRNA targets and no doubt affects their expression and metabolism, one might expect that the expression of HMGA1-p might have a pleiotropic effect. Thus, although the impact on HMGA1 mRNA and downstream INSR is clearly demonstrated in this paper, the apparent specificity of this effect remains an interesting observation that deserves further consideration.

In conclusion, our findings further highlight the functional role of pseudogenes in the regulation of mRNA stability/degradation in mammals. In addition, the data provide the first direct evidence that overexpression of the HMGA1-p pseudogene can give rise to human-specific disorders such as T2D. Further studies can now be initiated to elucidate the mechanistic basis for the aberrant overexpression of HMGA1-p expression in affected individuals.

\section{Methods}

Cells, IP/WB. All experiments with human cells were approved by the Comitato Etico Regione Calabria, Azienda Ospedaleria 'Mater Domini', Catanzaro.
EBV-transformed lymphoblasts were established from patients and from nondiabetic $(n=20)$ and diabetic $(n=27)$ individuals (Istituto G. Gaslini, Genova, Italy, Telethon Genetic Biobank Network, Project GTB07001A). All lymphoblastoid cell lines were maintained in RPMI 1640 medium supplemented with $15 \%$ fetal bovine serum and growth rates were similar in all cells. Human (HeLa and HEK-293) and mouse (3T3-L1) cell lines (ATCC) were maintained in Dulbecco's modified Eagle's medium with 10\% fetal bovine serum. 3T3-L1 fibroblasts were differentiated into 3T3-L1 adipocytes according to a standard protocol ${ }^{4}$. In studies of insulin signalling, 3T3-L1 cells were serum deprived for $3 \mathrm{~h}$ and treated with insulin for $10 \mathrm{~min}$ before preparation of whole-cell lysates. IP and WB of INSR, HMGA1 and Sp1 were performed on total cellular lysates or nuclear extracts from EBV lymphoblasts and human and mouse cultured cells ${ }^{3,4}$. Total and tyrosine-phosphorylated INSR and IRS1 were assayed in 3T3-L1 cells after IP of cell lysates using INSR $\beta$ and IRS 1 antibodies, followed by immunoblotting using an antiphosphotyrosine antibody. The antibodies used were anti-HMGA1 ${ }^{3,4}$; anti-INSR $\beta$, anti-Sp1, anti- $\beta$-actin (Santa Cruz Biotechnology); anti-phosphotyrosine and anti-IRS1 (Upstate Biotechnology).

RT-PCR, northern blot, and mRNA decay. Total cellular RNA was extracted from cells using an RNAqueous-4PCR kit and subjected to DNase treatment (Ambion). RNA levels were normalized against $18 \mathrm{~S}$ ribosomal RNA in each sample. Complementary DNA was synthesized from total RNA with a RETROscript first strand synthesis kit (Ambion) and used for PCR amplification. Northern blots and hybridizations were carried out following standard procedures. Probes for HMGA1 and HMGA1-p were obtained by amplifying the respective $5^{\prime}$-UTR non-homologous regions. Hybridization signals were quantified with Molecular Dynamics PhosphorImager and Imagequant software (Molecular Dynamics). For the half-life of HMGA1 mRNA, logarithmic-phase EBV lymphoblasts were exposed to $0.5 \mu \mathrm{g} \mathrm{ml}^{-1}$ of actinomycin D. RNA was extracted at 4-h intervals, cDNA was prepared and HMGA1 mRNA levels were measured by quantitative RT-PCR, using RPS9 mRNA as control. mRNA half-life was determined by regression analysis and visual determination of the point at which the best-fit line crossed the $50 \%$ intercept.

Transfections and ${ }^{125} \mathrm{I}-$ labelled insulin binding. pGL3 control vector, containing HMGA1 or HMGA1-p, was transfected into either HeLa, HEK-293 or 3T3-L1 cells, using LipofectAMINE 2000 reagent (Invitrogen), and HMGA1 and INSR mRNA and protein expression levels were assayed $48-96 \mathrm{~h}$ later. For siRNA experiments 100-200 pmol of siRNA targeted to HMGA1-p (Invitrogen) was transfected in EBV-transformed lymphoblasts $\left(4 \times 10^{6}\right.$ cells) using the diethylaminoethyl (DEAE) dextran method ${ }^{4}$, and nuclear extracts or total cellular lysates were prepared $72 \mathrm{~h}$ later. siRNA (100-200 pmol) was used to knock down $\alpha \mathrm{CP} 1$ in transfected HeLa cells $^{33}$. A nonspecific siRNA with a similar GC content (Dharmacon) and a nontargeting scrambled sequence (Santa Cruz Biotechnology) were used as a control. ${ }^{125} \mathrm{I}-$ insulin binding to EBV-transformed lymphoblasts treated with siRNA to HMGA1- $p$ was measured $72 \mathrm{~h}$ after transfection ${ }^{3,4}$.

EMSA and biotin pull-down assays. The $3^{\prime}$ end of both HMGA1 (H7) and HMGA1-p (H7-p) cDNA containing the appropriate T7 promoter sequence was generated by PCR and $1 \mu \mathrm{g}$ of PCR product was transcribed using the AmpliScribe T7-Flash Transcription Kit (Epicentre) with a portion of the UTP replaced by Biotin-16-UTP (Ambion). Biotin-labelled RNA probes ( $500 \mathrm{fmoles}$ each) were incubated with HeLa cytoplasmatic extracts and RNA-protein binding and pulldown assays were performed. Briefly, the RNA probe was incubated for $1 \mathrm{~h}$ with protein extract $(5 \mu \mathrm{g})$ in $1 \times$ binding buffer $(10 \mathrm{mM}$ Hepes, $40 \mathrm{mM} \mathrm{KCl}, 3.0 \mathrm{mM}$ $\mathrm{MgCl}_{2}, 1 \mathrm{mM}$ dithiothreitol, $5 \%$ glycerol, $1 \mu \mathrm{g}$ tRNA and $1.25 \mathrm{mg} \mathrm{ml}^{-1}$ heparin) at room temperature. The binding reaction was subsequently incubated with RNase A (10 ng $\mu \mathrm{l}^{-1}$, Qiagen) for $10 \mathrm{~min}$ and $1 \mu \mathrm{l}$ heparin $\left(50 \mathrm{mg} \mathrm{ml}^{-1}\right)$ was added to each reaction mixture. RNA-protein complexes were separated 10 min later on SDS-PAGE (6\%) and transferred to Hybond-N + membranes. Signal of RNA-protein binding was detected using the LightShift chemiluminescent kit (Pierce). FF1 antiserum antibody (against $\alpha \mathrm{CP} 1)^{34}$ was used in supershift experiments. RNA used as nonspecific competitor was an oligonucleotide of unrelated sequence (Supplementary Table S1) obtained by PCR using the AmpliScribe T7-Flash Transcription Kit, in the presence of T7 promoter sequence. RNA pull-down assays were carried out with $2 \mu \mathrm{g}$ of either $\mathrm{H} 7$ or H7-p biotinylated probe coupled to Streptavidin-coated M280 Dynabeads (Invitrogen). HeLa cell lysates (100 $\mu \mathrm{g})$ plus tRNA $(50 \mu \mathrm{g})$ were added to RNA beads and incubated for $30 \mathrm{~min}$ at $4{ }^{\circ} \mathrm{C}$. At the end of the incubation period, the supernatant was removed, the beads were washed three times with $100 \mu \mathrm{l}$ of hypotonic buffer, resuspended in $20 \mu \mathrm{l} 1 \times$ Laemmli lysis buffer and analysed by WB analysis using FF1 antiserum.

RNA IP assay. HMGA1 mRNA was detected by RT-PCR, after RNA IP, using anti- $\alpha$ CP1 antibody FF1. HeLa cells $\left(5 \times 10^{6}\right)$ were lysed in IP buffer $(50 \mathrm{mM}$ Tris- $\mathrm{HCl}, \mathrm{pH} 7.4 / 1 \%$ Nonidet P-40/0.5\% sodium deoxycholate/0.05\% SDS/1 mM EDTA/150 $\mathrm{mM} \mathrm{NaCl}$ ) containing protease inhibitors (Sigma) and $50 \mathrm{U} \mathrm{ml}^{-1}$ Superasin RNase inhibitor (Ambion), centrifuged at $12,000 \mathrm{~g}$ for $10 \mathrm{~min}$ at $4{ }^{\circ} \mathrm{C}$, after which the supernatant $(0.75 \mathrm{ml})$ was added to $10 \mu \mathrm{g}$ of FF1 antiserum antibody. After $2 \mathrm{~h}$ of incubation at room temperature, an equal volume of protein $\mathrm{A} / \mathrm{G}$ agarose beads was added and the mixture was incubated for an additional 
$2 \mathrm{~h}$. The protein A/G agarose-antibody-protein complexes were washed with IP buffer, bound RNAs were extracted using the RNAqueous-4PCR Kit and subjected to DNase treatment (Ambion); thereafter, HMGA1 mRNA was detected by RT-PCR.

The studies on humans were approved by the local ethics committee (Comitato Etico Regione Calabria, Azienda Ospedaleria 'Mater Domini', Catanzaro). We obtained informed consent from all individuals or from their parents.

Statistical analysis was performed using the Student's $t$-test. For all analyses, $P<0.05$ was considered significant.

DNA and RNA sequences are described in Supplementary Table S1.

\section{References}

1. Bustin, M. \& Reeves, R. High-mobility group proteins: architectural components that facilitates chromatin function. Prog. Nucleic Acids Res. 54, 35-100 (1996)

2. Cleynen, I. \& Van de Ven, W. J. The HMGA proteins: a myriad of functions. Int. J. Oncol. 32, 289-305 (2008).

3. Brunetti, A., Manfioletti, G., Chiefari, E., Goldfine, I. D. \& Foti, D. Transcriptional regulation of human insulin receptor gene by the high-mobility group protein HMGI-Y. FASEB J. 15, 492-500 (2001).

4. Foti, D., Iuliano, R., Chiefari, E. \& Brunetti, A. A nucleoprotein complex containing Sp1, C/EBP $\beta$, and HMGI-Y controls human insulin receptor gene transcription. Mol. Cell. Biol. 23, 2720-2732 (2003).

5. Foti, D. et al. Lack of the architectural factor HMGA1 causes insulin resistance and diabetes in humans and mice. Nat. Med. 11, 765-773 (2005).

6. Strichman-Almashanu, L. Z., Bustin, M. \& Landsman, D. Retroposed copies of the HMG genes: a window to genome dynamics. Genome Res. 13, 800-812 (2003).

7. Alberts, B., Johnson, A., Lewis, J., Raff, M., Roberts, K. \& Walter, P. in Molecular Biology of the Cell, 5th edn, 492-497 (Garland Science, London, 2007).

8. Borrmann, L., Wilkening, S. \& Bullerdiek, J. The expression of HMGA genes is regulated by their 3' UTR. Oncogene 20, 4537-4541 (2001).

9. Liebhaber, S. A. mRNA stability and the control of gene expression. Nucleic Acids Symp. Ser. 36, 29-32 (1997).

10. Rodgers, N. D., Wang, Z. \& Kiledjian, M. Regulated alpha-globin mRNA decay is a cytoplasmic event proceeding through $3^{\prime}$-to- $5^{\prime}$ exosome-dependent decapping. RNA 8, 1526-1537 (2002).

11. Waggoner, S. A. \& Liebhaber, S.A. Regulation of $\alpha$-globin mRNA stability. Exp. Biol. Med. 228, 387-395 (2003).

12. Vanin, E. F. Processed pseudogene: characteristics and evolution. Annu. Rev. Genet. 19, 253-272 (1985).

13. Balakirev, E. S. \& Ayala, F. J. Pseudogenes: are they 'junk' or functional DNA? Annu. Rev. Genet. 37, 123-151 (2003).

14. Goncalves, I., Duret, L. \& Mouchiroud, D. Nature and structure of human genes that generate retropseudogenes. Genome Res. 10, 672-678 (2000).

15. Harrison, P. M. et al. Molecular fossils in the human genome: identification and analysis of the pseudogene in chomosome 21 and 22. Genome Res. 12, 272-280 (2002).

16. Torrents, D., Suyama, M., Zdobnov, E. \& Bork, P. A genome-wide survey of human pseudogenes. Genome Res. 13, 2559-2567 (2003).

17. Khelifi, A., Duret, L. \& Mouchiroud, D. HOPPSIGEN: a database of human and mouse processed pseudogenes. Nucleic Acids Res. 33, D59-D66 (2005).

18. Zheng, D. et al. Pseudogenes in the ENCODE regions: consensus annotation, analysis of transcription, and evolution. Genome Res. 17, 839-851 (2007).

19. The ENCODE project Consortium. Identification and analysis of functional elements in $1 \%$ of the human genome by the ENCODE pilot project. Nature 447, 799-816 (2007).

20. Svensson, O., Arvestad, L. \& Lagergren, J. Genome-wide survey for biologically functional pseudogenes. PLoS Comp. Biol. 2, 358-369 (2006).

21. Sakai, H., Koyanagi, K. O., Imanishi, T. \& Gojobori, T. Frequent emergence and functional resurrection of processed pseudogenes in the human and mouse genomes. Gene 389, 196-203 (2007).
22. Korneev, S. A., Park, J. H. \& O'Shea, M. Neuronal expression of neural nitric oxide synthase (nNOS) protein is suppressed by an antisense RNA transcribed from a NOS pseudogene. J. Neurosci. 19, 7711-7720 (1999).

23. Hirotsune, S. et al. An expressed pseudogene regulates the messenger-RNA stability of its homologous coding gene. Nature 423, 91-96 (2003).

24. Gray, T. A., Wilson, A., Fortin, P. J. \& Nicholls, R. D. The putatively functional Mkrn1-pl pseudogene is neither expressed nor imprinted, nor does it regulate its source gene in trans. Proc. Natl Acad. Sci. USA 103, 12039-12044 (2006).

25. Duret, L., Chureau, C., Samain, S., Weissenbach, J. \& Avner, P. The Xist RNA gene evolved in eutherians by pseudogenization of a protein-coding gene. Science 312, 1653-1655 (2006).

26. Piehler, A. P. et al. The human ABC transporter pseudogene family: evidence for transcription and gene-pseudogene interference. BMC Genomics 9, 165 (2008).

27. Tam, O. H. et al. Pseudogene-derived small interfering RNAs regulate gene expression in mouse oocytes. Nature 453, 534-538 (2008).

28. Watanabe, T. et al. Endogenous siRNAs from naturally formed dsRNAs regulate transcripts in mouse oocytes. Nature 453, 539-543 (2008).

29. Zou, M. et al. Oncogenic activation of MAP kinase by BRAF pseudogene in thyroid tumors. Neoplasia 11, 57-65 (2009).

30. Bier, A. et al. Connexin 43 pseudogene in breast cancer cells offers a novel therapeutic target. Mol. Cancer. Ther. 8, 786-793 (2009).

31. Holcik, M. \& Liebhaber, S. A. Four highly stable eukaryotic mRNAs assemble 3 untranslated region RNA-protein complexes sharing cis and trans components. Proc. Natl. Acad. Sci. USA 94, 2410-2414 (1997).

32. Makeyev, A. V. \& Liebhaber, S. A. The poly(C)-binding proteins: a multiplicity of functions and a search for mechanisms. RNA 8, 265-278 (2002).

33. Waggoner, S. A., Johannes, G. J. \& Liebhaber, S. A. Depletion of the Poly $(C)$ binding proteins $\mathrm{CP} 1$ and $\mathrm{CP} 2$ from $\mathrm{K} 562$ cells Leads to $\mathrm{p} 53$-independent induction of Cyclin-dependent kinase inhibitor (CDKN1A) and G1 arrest. J. Biol. Chem. 284, 9039-9049 (2009).

34. Makeyev, A. V., Eastmond, D.L. \& Liebhaber, S. A. Targeting a KH-domain protein with RNA decoys. RNA 8, 1160-1173 (2002).

\section{Acknowledgments}

We are most grateful to F.S. Brunetti for his help in artwork preparation. We also thank A. Malta, T. Rossano, G. Ceravolo and G. Grandinetti for secretarial help. This work was supported by Telethon-Italy, Grant GGP04245, and MIUR, protocol 2004062059-002 Italy, to A. Brunetti.

\section{Author contributions}

E.C. conceived the study and contributed to the writing of the paper. S.I. and I.L.P. performed transfection assays; E.C. and F.P. conducted studies with EBV-transformed lymphoblasts; B.A. performed RNA-EMSA; M.F. prepared EBV-transformed lymphoblastoid cell lines from human blood samples; D.F. conducted studies on mRNA decay; S.A.L. contributed to the data analysis and revision of the paper. A.B. coordinated and supervised the study, analysed the data and wrote the paper. All authors discussed the results and commented on the paper.

\section{Additional information}

Accession numbers: HMGA1 mRNA variant 5: NM_145903; HMGA1-p: AC005041; HMGA1 mRNA variant 1: NM_145899; HMGA1: NT_007592.15.

Supplementary information accompanies this paper on http://www.nature.com/ naturecommunication

Competing financial interests: The authors declare no competing financial interests.

Reprints and permission information is available online at http://npg.nature.com/ reprintsandpermissions/

How to cite this article: Chiefari, E. et al. Pseudogene-mediated posttranscriptiona silencing of HMGA1 can result in insulin resistance and type 2 diabetes. Nat. Commun. 1:40 doi: $10.1038 /$ ncomms1040 (2010). 